\title{
Inflammatory mediators in the diagnosis and treatment of acute pancreatitis: pentraxin-3, procalcitonin and myeloperoxidase
}

\author{
Osman Simsek ${ }^{1}$, Ahmet Kocael ${ }^{1}$, Pınar Kocael ${ }^{1}$, Anıl Orhan ${ }^{1}$, Mahir Cengiz ${ }^{2}$, Huriye Balcı ${ }^{3}$, \\ Kenan Ulualp ${ }^{1}$, Hafize Uzun ${ }^{4}$
}

\author{
'Department of General Surgery, Cerrahpasa Medical Faculty, Istanbul University, \\ Istanbul, Turkey \\ ${ }^{2}$ Department of Internal Medicine, Cerrahpasa Medical Faculty, Istanbul University, \\ Istanbul, Turkey \\ ${ }^{3}$ Central Research Laboratory, Cerrahpasa Medical Faculty, Istanbul University, \\ Istanbul, Turkey \\ ${ }^{4}$ Department of Biochemistry, Cerrahpasa Medical Faculty, Istanbul University, \\ Istanbul, Turkey
}

Submitted: 2 September 2015

Accepted: 7 December 2015

Arch Med Sci 2018; 14, 2: 288-296

DOI: https://doi.org/10.5114/aoms.2016.57886

Copyright $\odot 2018$ Termedia \& Banach

\section{Abstract}

Introduction: Acute pancreatitis (AP) is the third most common gastrointestinal disease at hospital admission. The etiology and pathogenesis of this disease are not completely clear. Our study was intended to determine the systemic levels of pentraxin-3 (PTX-3), myeloperoxidase (MPO), procalcitonin (PCT), and C-reactive protein (CRP) as prognostic parameters in early stages of AP. We also determined the effects of treatment on PTX-3, MPO, PCT and CRP levels in AP.

Material and methods: The study group comprised 44 AP patients (22 male, 22 female; age: $49.3 \pm 16.9$ years) referred to our outpatient clinic. Additionally, our investigation included a control group of 30 healthy volunteers (18 male, 12 female; age: $50.8 \pm 12.6$ years).

Results: Leukocytes, glucose, aspartate aminotransferase (AST (SGOT)), alanine aminotransferase (ALT (SGPT)), alkaline phosphatase (ALP), total and direct bilirubin levels were significantly higher in the AP group $(p<0.05$, all). CRP, PTX-3, MPO and PCT were considerably higher in the AP group $(p<0.001$, all), and after treatment, CRP, PTX-3, MPO and PCT levels were significantly lower $(p<0.001$, all).

Conclusions: Our findings indicated that the CRP, PTX-3, MPO and PCT levels increase in patients with AP and hence these indicators can be used as diagnostic factors to predict inflammation severity in AP. It was revealed that after treatment, there were significant reductions in biomarker levels. However, further research is needed in order to understand how these biomarkers can help to monitor inflammatory responses in AP.

Key words: acute pancreatitis, pentraxin-3, procalcitonin, myeloperoxidase.

\section{Introduction}

Acute pancreatitis (AP) is a common acute abdominal disease. Inflammatory mediators play an essential role in the development of acute pancreatitis and in systemic complications, which are the dominant reasons

\author{
Corresponding author: \\ Ahmet Kocael \\ Department of \\ General Surgery \\ Cerrahpasa Medical Faculty \\ Istanbul University \\ 34098 Istanbul, Turkey \\ Fax: +90 02124143000 \\ E-mail: \\ akocael147@yahoo.com
}


for patient mortality. The exact pathways of the various etiologies that trigger pancreatitis are still unclear; however, once the attack is triggered, the inflammatory processes and repair mechanisms are well-understood [1].

Acute phase proteins are helpful molecules for detecting the presence of inflammatory disorders. C-reactive protein (CRP) is a component of the pentraxin superfamily along with pentraxin-3 (PTX-3), a protein composed by a long characteristic N-terminal domain coupled to the C-terminal PTX domain, which is emerging as an important player in immunity and inflammation [2].

Myeloperoxidase (MPO) is an important heme enzyme, mainly released by activated neutrophils, characterized by powerful proinflammatory properties. Myeloperoxidase is associated with "classical" inflammatory responses in various inflammatory diseases. Recent evaluations have shown that MPO has been a useful risk marker and diagnostic tool in both patients and experimental animals with AP [3].

Procalcitonin (PCT) has a molecular weight of $14.5 \mathrm{kDa}$ and is the inactive 116 amino acid pro-peptide of the biologically active hormone calcitonin. Parafollicular cells (C cells) of the thyroid and neuroendocrine cells of the lung and the intestine produce procalcitonin as a response to invasions by pathogenic bacteria, fungi, and some parasites. Procalcitonin is known for its sensitivity to bacterial infections and may help to differentiate bacterial infections from non-bacterial ones [4].

Procalcitonin has been investigated to predict the development of inflammation in AP. However, the clinical effectiveness of this parameter in AP is controversial [5-15]. To adequately evaluate the accuracy of inflammatory markers, we determined the systemic levels of PTX-3, MPO, PCT, and CRP as prognostic markers in early phases of AP. To our knowledge, this is the first study to link the properties of treatments with the inflammatory response in patients with AP. Our aim in this study was also to investigate the effects of treatment on PCT, PTX-3, MPO and CRP levels in AP.

\section{Material and methods}

\section{Study population}

The study group consisted of 44 patients who were admitted to our outpatient clinic over a 6-month period from June to December 2014 and diagnosed with acute pancreatitis (22 male, 22 female; age: $49.3 \pm 16.9$ years). We also created a control group consisting of 30 healthy volunteers (18 male, 12 female; age: $50.8 \pm 12.6$ years).

Acute pancreatitis was defined by at least two of the following criteria: 1) epigastric abdominal pain; 2) elevated serum amylase and/or lipase values (which exceeded three times the upper limit of normal or more); and 3) a computed tomography (CT) scan demonstrating characteristic changes of AP. The severity of AP was determined according to the most recently revised Atlanta Classification [16, 17].

We used computed tomography in selected patients if the diagnosis was not certain, in patients who did not show significant improvements in their clinical status after 48 to $72 \mathrm{~h}$, and patients who had atypical and complex complaints at admission.

After the correct diagnosis of AP was established by clinical features and laboratory results, medical treatment was planned for patients. Standard treatments for patients were intravenous resuscitation, electrolyte replacement and analgesia. Nasogastric decompression was planned for patients describing nausea and vomiting in order to prevent aspiration to lungs. We used routine antibiotic prophylaxis if there was an extrapancreatic infection such as cholangitis, bacteremia, urinary tract infections, or pneumonia, when sterile necrosis converted to infected necrosis, or when we failed to cure pancreatic or extra-pancreatic necrosis within 7 to 10 days after hospital admission.

We used total parenteral nutrition for nutritional support oxygen support in order to prevent hypoxia. If possible, we performed cholecystectomies of patients in the same hospital admission when their clinical features were suitable. An endoscopic sphincterotomy was performed when biliary tract drainage was required.

Blood samples were taken before and after treatment of the patients diagnosed with acute pancreatitis. All markers were measured again at least 1 week after discharge from hospital. Subjects with signs or symptoms of inflammatory bowel disease, connective tissue disorders (rheumatoid arthritis, systemic lupus erythematosus, scleroderma, Sjögren's syndrome, etc.), atherosclerotic vascular disease, thyroid disease (hypo/ hyperthyroidism), malignancy and chronic liver and/or kidney diseases were excluded. Patients using anti-inflammatory (corticosteroids, azathioprine, and non-steroidal anti-inflammatory drugs) and immunomodulatory agents within the previous 4 weeks were also excluded. Patients taking drugs that could lead to acute pancreatitis were excluded from the study.

\section{Informed consent}

The permission for sample collection was granted by Istanbul University, Cerrahpasa Medicine Faculty Ethics Committee, and the procedure was executed according to the requirements of the Declaration of Helsinki. All of the patients were fully briefed on the study procedures before they gave their consent. 


\section{Sample collection and measurements}

Blood samples were acquired in tubes containing EDTA (for PTX-3 and PCT), lithium heparin (for MPO) and anticoagulant-free tubes in the morning after $12-14 \mathrm{~h}$ of fasting. Centrifugation at $2500 \times \mathrm{g}$ for 5 min was performed; the plasma and serum were separated within $30 \mathrm{~min}$. Each sample was split into four aliquots, and the samples were stored at $-80^{\circ} \mathrm{C}$ until biochemical analysis.

\section{Measurement of plasma PTX-3 concentrations}

Plasma PTX-3 levels were measured using a commercially available sandwich ELISA (Human Pentraxin 3, Hycult Biotechnology, HP9039; Uden, The Netherlands). The coefficients of intra- and inter-assay variation were $4.0 \%(n=10)$ and $5.3 \%$ $(n=10)$, respectively.

\section{Measurement of plasma PCT concentrations}

The plasma PCT levels were calculated by an enzyme-linked immunoassay using a commercially available kit (Uscn Life Science Inc., ELISA Kit, Cat. No: SEA689Hu, USA) according to the manufacturer's directions. The coefficients of intra- and inter-assay variation were $5.1 \%(n=10)$ and $6.1 \%$ $(n=10)$, respectively.

\section{Measurements of plasma MPO concentrations}

Plasma MPO levels were measured using a commercially available enzyme-linked immunosorbent assay kit (Enzo Life Sciences AG, Industriestrasse 17, Switzerland) according to the manufacturer's directions. The coefficients of intra- and inter-assay variation were $4.3 \%(n=10)$ and $5.7 \%$ $(n=10)$, respectively.

The serum CRP levels were measured using a nephelometric method (Immage 800 Beckman Coulter). The other biochemical parameters were calculated by routine methods with commercial kits.

\section{Statistical analysis}

The normal distribution of the data was tested using the 1-sample Kolmogorov-Smirnov test. All statistical comparisons were calculated and compared using the two-sided Student's $t$-test. The unpaired $t$-test was used to confirm the non-parametric Mann-Whitney $U$ test. An analysis of variance (ANOVA) was used to compare multigroup means. The following post-hoc evaluation was made using the Bonferroni method. Categorical variables were compared using a $\chi^{2}$ test or Fisher's exact test for small samples. The values exhibiting continuity were presented as a mean or standard deviation. Pearson's correlation was used for numerical data. Spearman's correlation was used for nominal data. To evaluate the diagnostic accuracy, we performed receiver operating characteristic (ROC) curve analysis. The area under the ROC curve (AUC) was then estimated with a 95\% confidence interval. A multivariate logistic regression model was performed to determine the effect of independent risk factors for acute pancreatitis patients and controls. Values where $p<0.05$ were considered statistically significant. Statistical analyses were performed using SPSS 20.0 for Windows (SPSS Inc., Chicago, IL, USA).

\section{Results}

The demographic, clinical and laboratory characteristics of the AP and control groups are summarized in Table I. Age, gender distribution, body mass index (BMI), and creatinine were not significantly different between the groups. Comorbidities such as hypertension and diabetes mellitus were similar in both groups. Alcohol and tobacco consumption was similar between the groups. Leukocyte, glucose, aspartate aminotransferase (AST), alanine aminotransferase (ALT), alkaline phosphatase (ALP) and total and direct bilirubin levels were significantly higher in the AP group ( $p<0.05$, all). CRP, PTX-3, PCT and MPO levels were very significantly higher in the AP group ( $p<0.001$, all).

The demographic, clinical and laboratory aspects of all of the groups are summarized in Table II. The leukocyte and hematocrit levels were significantly higher before treatment in the AP group ( $p<0.001$ and $p<0.001$, respectively). As expected, the glucose, total bilirubin, AST, ALT, ALP and amylase levels before treatment were significantly higher in the AP group compared to the control group $(p<0.001$, all). The CRP, PTX-3, PCT and MPO levels were significantly higher before treatment in the AP group compared to the control group ( $p<0.001$, all).

The acute pancreatitis group was divided into three groups according to etiology of acute pancreatitis as gallstones, alcohol, and other. The etiology of acute pancreatitis was gallstones 32 (72.7\%), alcohol 3 (6.8\%), and other 9 (20.5\%) patients. Amylase, CRP, PTX-3, PCT and MPO levels were not significantly different between the groups.

All of the results were analyzed according to the severity of disease, which was classified as either mild or severe. In the AP group, 28 patients had mild disease and 16 patients had severe disease. There was no statistical variation among the groups when comparing the amylase, CRP, PTX-3, PCT and MPO levels. After treatment, the CRP, PTX-3, MPO and PCT levels were significantly lower $(p<0.001$, all).

The PTX-3 levels were significantly positively correlated with the leukocyte $(r=0.429$, 
$p=0.004)$, PCT $(r=0.315, p=0.037)$ and CRP $(r=0.411, p=0.006)$ levels in the AP group. The PCT was significantly positively correlated with the MPO $(r=0.322, p=0.033)$ and CRP $(r=$ $0.826, p<0.001)$ levels in the AP group. The MPO activity was significantly positively correlated with $\operatorname{CRP}(r=0.384, p=0.01)$ in the AP group (Figure 1 ). The PTX-3 levels were significantly positively correlated with the leukocyte $(r=0.315, p=0.038)$ and PCT ( $r=0.387, p=0.009)$ levels in the AP group after treatment.

The results of multivariate logistic regression analysis including $\beta$ and $t$ value for each of the biochemical parameters are presented for the acute pancreatitis group in Table III.

To test the power of the amylase, leukocyte, CRP, PTX-3, PCT and MPO levels for distinguishing between the AP and control groups, ROC analysis was performed. ROC curves yielded an area under the curve of $0.937(p<0.001)$ for amylase, 0.800 $(p<0.001)$ for leukocytes, $0.979(p<0.001)$ for CRP, $1.00(p<0.001)$ for PTX-3, $1.00(p<0.001)$ for PCT and $0.855(p<0.001)$ for MPO in the diagnosis of AP (Figure 2, Table IV).

\section{Discussion}

Acute pancreatitis consists of a complex chain reaction wherein acinar cell damage leads to a local and systemic inflammatory response in experimental and human acute pancreatitis [1, 18]. We observed that in subjects with AP, the CRP, PTX-3, MPO and PCT levels were significantly and consistently higher than in the control group, and after

Table I. Demographic, clinical and laboratory characteristics of the acute pancreatitis and control groups

\begin{tabular}{|c|c|c|c|}
\hline Parameter & Acute pancreatitis $(n=44)$ & Controls $(n=30)$ & $P$-value \\
\hline Sex (female/male) & $22 / 22$ & $12 / 18$ & 0.479 \\
\hline Age [years] & $49.3 \pm 16.9$ & $50.8 \pm 12.6$ & 0.674 \\
\hline BMI $\left[\mathrm{kg} / \mathrm{m}^{2}\right]$ & $27.8 \pm 3.7$ & $26.1 \pm 4.1$ & 0.069 \\
\hline Smoking & $9(20.5 \%)$ & $4(13.3 \%)$ & 0.541 \\
\hline Alcohol use & $3(6.8 \%)$ & $2(6.7 \%)$ & 0.980 \\
\hline Hypertension & $20(45.5 \%)$ & $10(33.3 \%)$ & 0.342 \\
\hline Diabetes mellitus & $13(29.5 \%)$ & $8(26.7 \%)$ & 0.787 \\
\hline Ranson's score & $1.9 \pm 1.3$ & & \\
\hline \multicolumn{4}{|l|}{ CT severity index: } \\
\hline Mild & $28(63.6 \%)$ & & \\
\hline Severe & $16(36.4 \%)$ & & \\
\hline Leukocytes $\left[\mathrm{mm}^{3}\right]$ & $10.743 \pm 4.255$ & $6.669 \pm 1.395$ & $<0.001$ \\
\hline Hematocrit (\%) & $39.9 \pm 4.9$ & $39.3 \pm 1.6$ & 0.516 \\
\hline Glucose [mg/dl] & $137.9 \pm 57.9$ & $91.3 \pm 15.9$ & $<0.001$ \\
\hline Creatinine [mg/dl] & $0.79 \pm 0.2$ & $0.83 \pm 0.2$ & 0.451 \\
\hline Total bilirubin [mg/dl] & $2.7 \pm 3.6$ & $0.5 \pm 0.2$ & 0.001 \\
\hline Direct bilirubin [mg/dl] & $1.8 \pm 2.9$ & $0.3 \pm 0.1$ & 0.005 \\
\hline AST [U/l] & $147.2 \pm 169$ & $16.4 \pm 5.1$ & $<0.001$ \\
\hline ALT [U/l] & $188.5 \pm 248.1$ & $14.5 \pm 6.4$ & $<0.001$ \\
\hline ALP [U/l] & $181.8 \pm 147.8$ & $59.6 \pm 13.8$ & $<0.001$ \\
\hline Amylase [U/I] & $1580 \pm 1614$ & $51.2 \pm 13.5$ & $<0.001$ \\
\hline CRP $[\mathrm{mg} / \mathrm{dl}]$ & $24.9 \pm 30.8$ & $1.6 \pm 0.7$ & $<0.001$ \\
\hline Procalcitonin [pg/ml] & $175.1 \pm 93.1$ & $11.8 \pm 4.9$ & $<0.001$ \\
\hline Pentraxin-3 [pg/ml] & $1846 \pm 1155$ & $341.5 \pm 99.7$ & $<0.001$ \\
\hline Myeloperoxidase [ng/m] & $79.8 \pm 61.2$ & $34.5 \pm 10.2$ & $<0.001$ \\
\hline
\end{tabular}


treatment, the CRP, PTX-3, MPO and PCT levels were significantly lower. Meanwhile, PTX-3 levels were positively correlated with the leukocyte, PCT and CRP levels in the AP group. PTX-3 and PCT had the highest sensitivity and specificity. Nevertheless, information regarding the pathogenesis of inflammation in AP was not evident; our findings suggest that systemic inflammation takes place both severely and moderately in AP. PTX-3 and PCT may be useful for early estimation of the severity of AP. PTX-3 and PCT are the standard for a fast, reliable, and cost-effective assessment of severity of AP. Additionally, systemic PTX-3 and PCT levels may be used for better evaluation of clinical grade, as well as independent of grade, for the purpose of routine clinical laboratory analysis.

Pentraxins are among the main acute phase reactants. Pentraxin-3 is synthesized in response to proinflammatory stimuli. We found that the CRP and PTX-3 levels in the AP group were consistently higher than in the control patients, and these potential biomarkers were significantly decreased after treatment. To compare the performance of PTX-3 with an established biomarker in AP, we

Table II. Demographic, clinical and laboratory characteristics of all groups

\begin{tabular}{|c|c|c|c|c|}
\hline Parameter & $\begin{array}{l}\text { Pre-treatment acute } \\
\text { pancreatitis group } \\
\qquad(n=44)\end{array}$ & $\begin{array}{l}\text { Post-treatment acute } \\
\text { pancreatitis group } \\
\qquad(n=44)\end{array}$ & $\begin{array}{l}\text { Control group } \\
\quad(n=30)\end{array}$ & $P$-value \\
\hline Sex (female/male) & $22 / 22$ & $22 / 22$ & $12 / 18$ & 0.639 \\
\hline Age [years] & $49.3 \pm 16.9$ & $49.3 \pm 16.9$ & $50.8 \pm 12.6$ & 0.916 \\
\hline BMI $\left[\mathrm{kg} / \mathrm{m}^{2}\right]$ & $27.8 \pm 3.7$ & $27.8 \pm 3.7$ & $26.1 \pm 4.1$ & 0.104 \\
\hline Smoking & 9 (20.5\%) & 9 (20.5\%) & $4(13.3 \%)$ & 0.688 \\
\hline Alcohol use & $3(6.8 \%)$ & $3(6.8 \%)$ & $2(6.7 \%)$ & 0.996 \\
\hline Hypertension & $20(45.5 \%)$ & $20(45.5 \%)$ & $10(33.3 \%)$ & 0.510 \\
\hline Diabetes mellitus & $13(29.5 \%)$ & $13(29.5 \%)$ & $8(26.7 \%)$ & 0.956 \\
\hline Leukocytes $\left[\mathrm{mm}^{3}\right]$ & $10743 \pm 4255$ & $7954 \pm 3241$ & $6669 \pm 1395$ & $<0.001^{a}$ \\
\hline Hematocrit (\%) & $39.9 \pm 4.9$ & $35.3 \pm 4.3$ & $39.3 \pm 1.6$ & $<0.001^{b}$ \\
\hline Glucose [mg/dl] & $137.9 \pm 57.9$ & $112.6 \pm 40.3$ & $91.3 \pm 15.9$ & $<0.001^{c}$ \\
\hline Creatinine [mg/dl] & $0.79 \pm 0.2$ & $0.77 \pm 0.3$ & $0.83 \pm 0.2$ & 0.580 \\
\hline Total bilirubin [mg/dl] & $2.7 \pm 3.6$ & $0.6 \pm 0.4$ & $0.5 \pm 0.2$ & $<0.001^{d}$ \\
\hline Direct bilirubin [mg/dl] & $1.8 \pm 2.9$ & $0.5 \pm 0.3$ & $0.3 \pm 0.1$ & $0.003^{e}$ \\
\hline AST [U/I] & $147.2 \pm 169$ & $33.3 \pm 23.8$ & $16.4 \pm 5.1$ & $<0.001^{f}$ \\
\hline $\operatorname{ALT}[\mathrm{U} / \mathrm{I}]$ & $188.5 \pm 248.1$ & $41.1 \pm 28.5$ & $14.5 \pm 6.4$ & $<0.001^{\mathrm{g}}$ \\
\hline $\mathrm{ALP}[\mathrm{U} / \mathrm{I}]$ & $181.8 \pm 147.8$ & $115.7 \pm 68.1$ & $59.6 \pm 13.8$ & $<0.001^{h}$ \\
\hline Amylase [U/I] & $1580 \pm 1614$ & $132.9 \pm 206.9$ & $51.2 \pm 13.5$ & $<0.001^{\prime}$ \\
\hline CRP $[\mathrm{mg} / \mathrm{dl}]$ & $24.9 \pm 30.8$ & $21.1 \pm 14.7$ & $1.6 \pm 0.7$ & $<0.001^{j}$ \\
\hline Procalcitonin [pg/ml] & $175.1 \pm 93.1$ & $89.4 \pm 11.7$ & $11.8 \pm 4.9$ & $<0.001^{k}$ \\
\hline Pentraxin-3 [pg/ml] & $1846 \pm 1155$ & $1031 \pm 544$ & $341.5 \pm 99.7$ & $<0.001^{1}$ \\
\hline Myeloperoxidase [ng/ml] & $79.8 \pm 61.2$ & $37.1 \pm 19.3$ & $34.5 \pm 10.2$ & $<0.001^{\mathrm{m}}$ \\
\hline
\end{tabular}

$A L P$ - alkaline phosphatase, ALT - alanine transaminase, AST - aspartate aminotransferase, $B M I-$ body mass index, CRP - C-reactive protein, ${ }^{a}$ Acute pancreatitis and post-treatment AP groups, $p<0.001$; acute pancreatitis and control groups, $p<0.001 .{ }^{b} A c u t e ~ p a n c r e a t i t i s$ and post-treatment AP groups, $p<0.001$; post-treatment AP and control groups, $p<0.001$. $^{c}$ Acute pancreatitis and post-treatment AP groups, $p=0.024$; acute pancreatitis and control groups, $p<0.001$. ${ }^{d}$ Acute pancreatitis and post-treatment AP groups, $p<0.001 ;$ acute pancreatitis and control groups, $p<0.001 .{ }^{e}$ Acute pancreatitis and post-treatment AP groups, $p=0.015$; acute pancreatitis and control groups, $p=0.007$. ${ }^{f}$ Acute pancreatitis and post-treatment AP groups, $p<0.001$; acute pancreatitis and control groups, $p<0.001 .{ }^{g} A c u t e$ pancreatitis and post-treatment AP groups, $p<0.001$; acute pancreatitis and control groups, $p<0.001 .{ }^{h}$ Acute pancreatitis and posttreatment AP groups, $p=0.007$; acute pancreatitis and control groups, $p<0.001$. 'Acute pancreatitis and post-treatment AP groups, $p<0.001$; acute pancreatitis and control groups, $p<0.001$. $^{j}$ Acute pancreatitis and post-treatment AP groups, $p<0.001 ;$ acute pancreatitis and control groups, $p<0.001 .{ }^{k}$ Acute pancreatitis and post-treatment AP groups, $p<0.001$; acute pancreatitis and control groups, $p<0.001$; post-treatment AP and control groups, $p<0.001$.' Acute pancreatitis and post-treatment AP groups, $p<0.001$; acute pancreatitis and control groups, $p<0.001$; post-treatment $A P$ and control groups, $p=0.001 .{ }^{m}$ Acute pancreatitis and post-treatment AP groups, $p<0.001$; acute pancreatitis and control groups, $p<0.001$ 
A

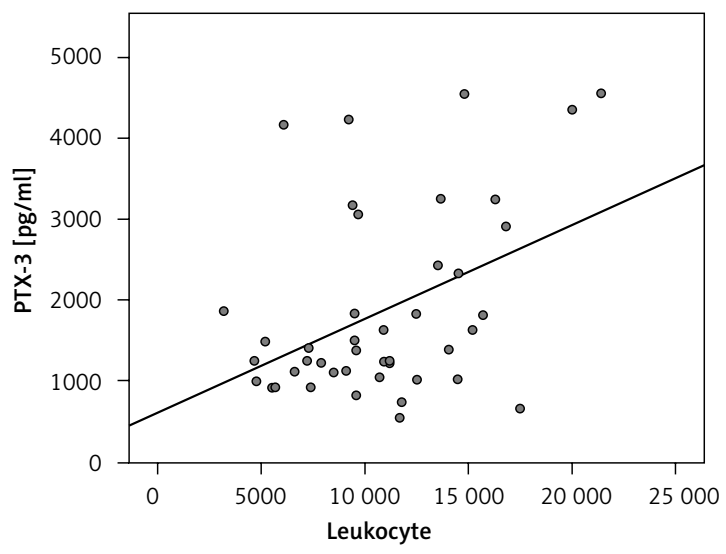

C

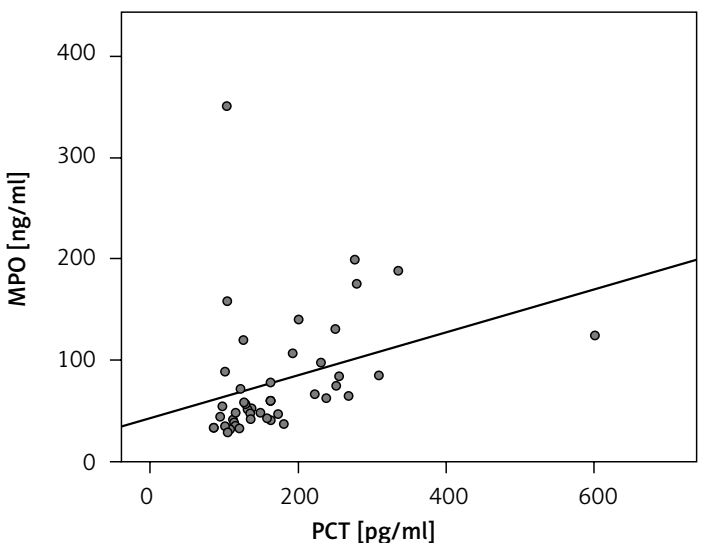

B

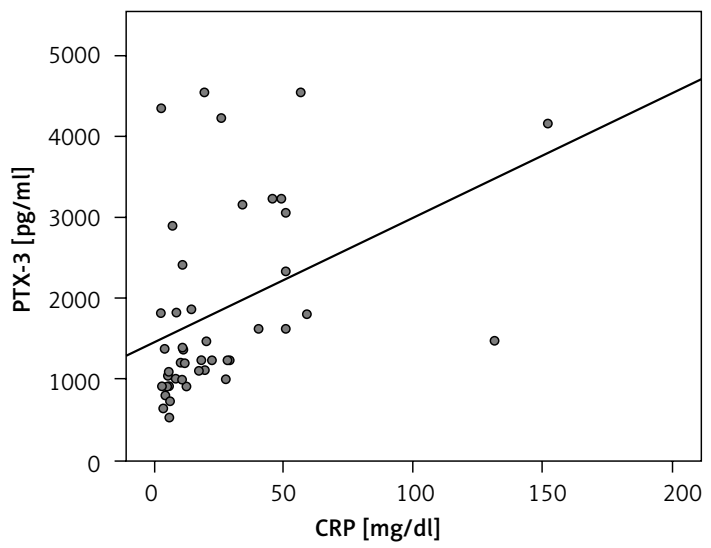

D

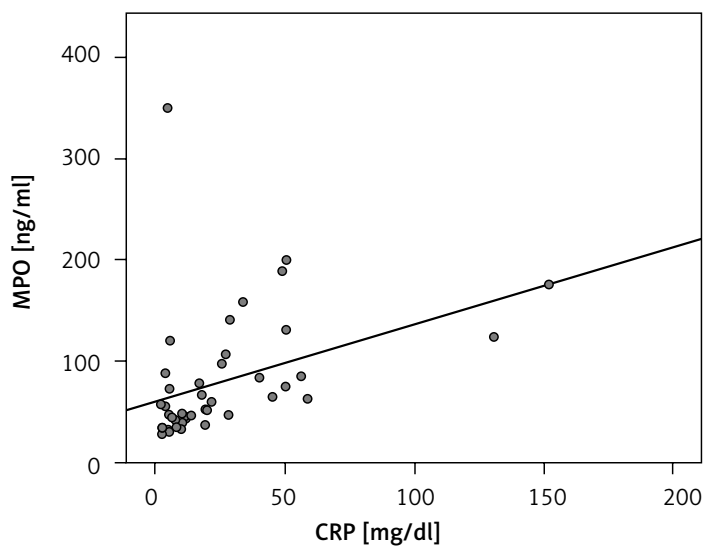

Figure 1. Relationship between PTX-3 and leukocyte levels (A), PTX-3 and CRP levels (B), MPO and PCT levels (C) and MPO and CRP levels (D) in the acute pancreatitis group

compared PTX-3 with CRP in the patients. The levels of PTX-3 and CRP correlated significantly. Using ROC curve analysis, PTX-3 (cut-off value, AUC) showed a significantly better positive predictive value than CRP. We demonstrated that PTX-3 may be a better biomarker than CRP for monitoring inflammatory responses in AP. Kusnierz-Cabala et al. [19] investigated the pattern of changes in PTX-3 concentration in the early stages of AP, and its peak values are achieved earlier compared to CRP. Meryk et al. [20] showed that in the early phase of AP, the concentrations of PTX-3 reached a maximum level earlier than CRP, allowing dis- tinctions among mild, moderate or severe AP on the first day of the disease. Our and other studies $[19,20]$ confirm that measurements of serum PTX-3 may be valuable for predicting the severity of AP.

Myeloperoxidase is a crucial enzyme for normal neutrophil function, and it is mainly released by activated neutrophils characterized by powerful proinflammatory properties. Myeloperoxidase release leads to tissue damage in clinical and experimental studies of AP. In the current study, the plasma MPO activity was higher in the AP group and was decreased after treatment. The MPO ac-

Table III. Logistic regression analysis results between the acute pancreatitis and control groups

\begin{tabular}{|lccc|}
\hline Parameter & $\boldsymbol{\beta}$ & $\boldsymbol{t}$ & $\boldsymbol{P}$-value \\
\hline Amylase & 0.171 & 2.312 & 0.024 \\
\hline CRP & -0.505 & -4.018 & $<0.001$ \\
\hline Procalcitonin & 0.903 & 6.698 & $<0.001$ \\
\hline Pentraxin-3 & 0.253 & 2.579 & 0.012 \\
\hline Myeloperoxidase & 0.107 & 1.444 & 0.153 \\
\hline Leukocyte & -0.023 & -0.266 & 0.791 \\
\hline
\end{tabular}


A

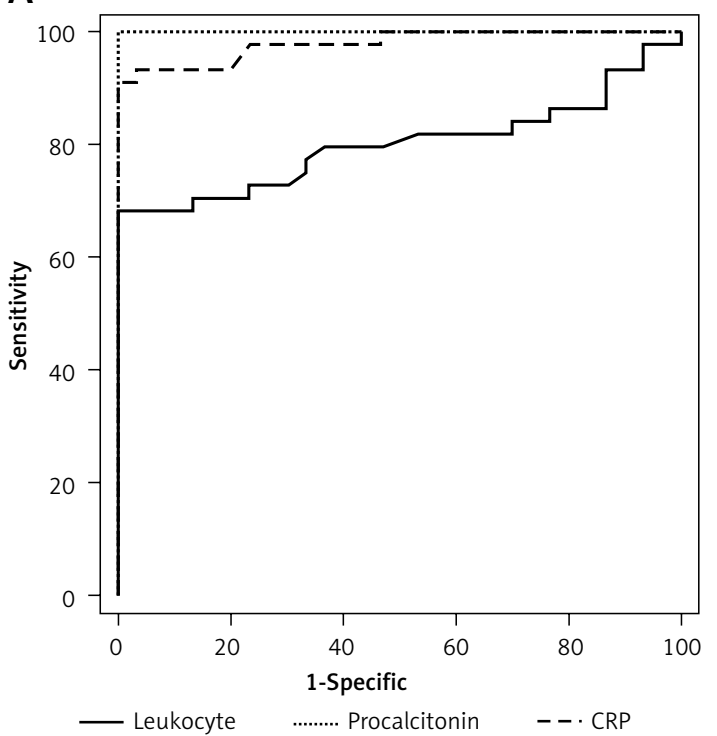

B

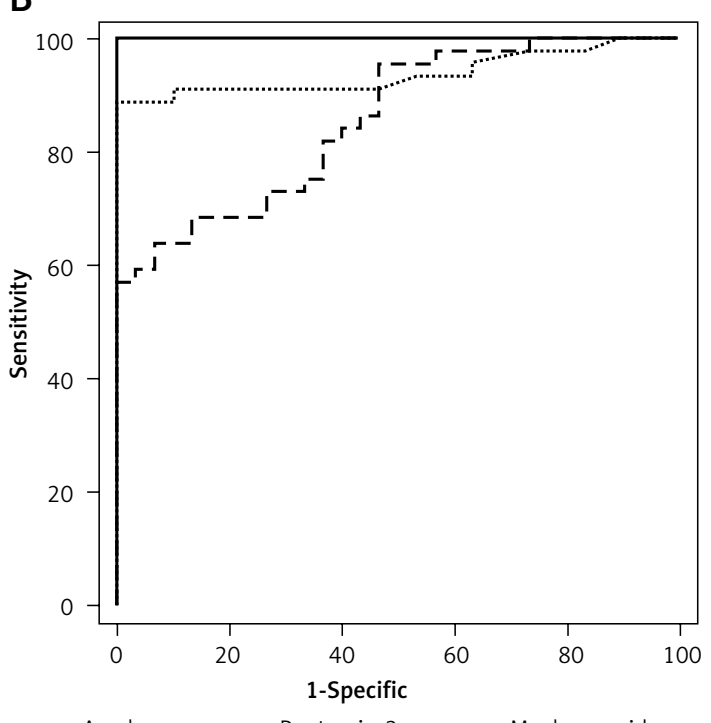

Figure 2. To test the power of amylase, leukocytes, CRP, PTX-3, PCT and MPO for distinguishing between acute pancreatitis and control groups

Table IV. Comparison of amylase, leukocyte, CRP, pentraxin-3, procalcitonin and myeloperoxidase levels using ROC analysis

\begin{tabular}{|lccccc|}
\hline Parameter & Sensitivity (\%) & Specificity (\%) & AUC & Cut-off & P-value \\
\hline Amylase & 90.9 & 90 & 0.937 & 68 & $<.001$ \\
\hline Leukocytes & 77.3 & 66.7 & 0.800 & 7250 & 2.6 \\
\hline CRP & 93.2 & 93.3 & 0.979 & 531.4 & $<0.001$ \\
\hline Pentraxin-3 & 100 & 100 & 1.00 & 1.00 & $54.8<0.001$ \\
\hline Procalcitonin & 100 & 100 & 0.855 & 42.6 & $<.001$ \\
\hline Myeloperoxidase & 72.7 & 70 & & $<.001<$ \\
\hline
\end{tabular}

$A U C$ - area under curve, $C R P-C$-reactive protein.

tivity was positively correlated with CRP in the AP group. Our data suggest that MPO may be beneficial in treating this type of inflammatory condition. Chooklin [21] reported that patients suffering from severe AP had the highest level of MPO on the first day. After 3 days, test results from all of the patients suffering from necrotizing pancreatitis showed a decrease in MPO blood levels. Pancreatitis-associated lung injury and purulent complications were associated with the increased MPO levels. The blood MPO level is dependent on the severity of AP. Park et al. [22] reported that patients suffering from mild pancreatitis had lower mean plasma levels of MPO compared to patients with severe pancreatitis. Plasma MPO levels showed a close association with the tomography severity index, but there was no significant relationship with serum CRP. The increase in MPO levels was more significant in severe pancreatitis compared to mild pancreatitis. Chooklin [21] noted that patients suffering from severe lung injury showed higher MPO activity in the blood, but MPO activity still represented the circumstances taking place in the lung parenchyma in AP. In patients, MPO activity regressed notably in the lungs due to a decreased concentration of neutrophils, which produce this enzyme. This is why evaluating MPO levels in patients with acute respiratory distress syndrome (ARDS) or acute lung injury (ALI) is key. Myeloperoxidase augments the damage to the alveolar-capillary membrane and stimulates lung injury. Correlation analysis has shown that in the development of respiratory dysfunction without ALI symptoms, the concentration of MPO correlates with serum levels of IL-8. Despite this, MPO is highly immunomodulatory. It is the authors' intention to relate multiple aspects of an enzyme that plays many known, as well as unrecognized, functions in inflammation [23].

Early assessment of the severity and etiology of AP is essential for management of the disease. Numerous biomarkers have been studied as potential early predictors of the severity of AP in order to plan an optimal treatment without com- 
plications. Few studies have researched the use of PCT in AP [5-15]. In the present study, PCT levels were significantly higher before treatment of AP compared to the control group. Until now, conventional treatments for AP have mainly been anti-inflammatory therapies which reduced PCT after treatment. There are some reports emphasizing the possible mechanisms of enhanced production of PCT. Modrau et al. [24] reported that PCT offered minimal additional value for the early assessment of the severity and etiology of AP. C-reactive protein is found to be a reliable prognostic marker at the 48-hour mark, while ALT was the best marker to suggest a biliary etiology. Rau et al. [25] investigated the value of PCT for identifying patients at risk of developing pancreatic infections in severe AP. In contrast to CRP, PCT concentrations showed a remarkable escalation in patients with pancreatic infections, and the concentration was associated with multiorgan dysfunction syndrome (MODS) requiring surgery $(n=10)$ or resulting in fatalities $(n=8)$ early after the onset of symptoms. The monitoring of PCT allows a quick and trustworthy evaluation of clinically relevant pancreatic infections and overall prognosis in AP. This single test parameter significantly contributes to improved stratification of patients at risk of developing major complications. Our study showed that the serum PCT was a major predictor of AP, and these results were consistent with our previous report [8, 24-33]. Plasma PCT levels may be used to suggest the clinical stage of AP using routine clinical laboratory analysis. Similarly, increased PCT levels in AP may indicate the early stages of a bacterial infection.

Our findings indicated that CRP, PTX-3, MPO and PCT levels increase in patients with AP and that they can be used as diagnostic factors to predict the severity of inflammation in AP. It was found that after treatment, there were significant reductions in biomarker levels. Our study is the first to document a significant decrease in CRP, PTX-3, MPO and PCT levels after treatment of patients with AP. In the future, PTX-3 and PCT may implemented in hospitals and used on a routine basis. There was no significant difference between the groups (mild and severe) when comparing amylase, CRP, PTX-3, PCT and MPO levels. Consequently, the effect of conventional therapeutic approaches on early changes in inflammatory mediator release should be evaluated shortly after clinical symptoms develop. Despite these difficulties, studies are still very effective in finding new markers to predict the severity of AP. More clinical and experimental research is needed to understand how these biomarkers can help monitor inflammation in AP.

\section{Conflict of interest}

The authors declare no conflict of interest.

\section{References}

1. Bhatia M, Brady M, Shokuhi S, Christmas S, Neoptolemos JP, Slavin J. Inflammatory mediators in acute pancreatitis. J Pathol 2000; 190: 117-25.

2. Bonacina F, Baragetti A, Catapano AL, Norata GD. Long pentraxin 3: experimental and clinical relevance in cardiovascular diseases. Mediators Inflamm 2013; 2013 : 725102.

3. Rayner BS, Love DT, Hawkins CL. Comparative reactivity of myeloperoxidase-derived oxidants with mammalian cells. Free Radic Biol Med 2014; 71: 240-55.

4. Schuetz P, Albrich W, Mueller B. Procalcitonin for diagnosis of infection and guide to antibiotic decisions: past, present and future. BMC Med 2011; 9: 107.

5. Brunkhorst FM, Eberhard OK, Brunkhorst R. Early identification of biliary pancreatitis with procalcitonin. Am J Gastroenterol 1998; 93: 1191-2.

6. Rau B, Steinbach G, Baumgart K, Gansauge F, Grünert A, Beger $\mathrm{H}$. The clinical value of procalcitonin in the prediction of infected necrois in acute pancreatitis. Intensive Care Med 2000; 26: S159-64.

7. Gurda-Duda A, Kusnierz-Cabala B, Nowak W, Naskalski JW, Kulig J. Assessment of the prognostic value of certain acute-phase proteins and procalcitonin in the prognosis of acute pancreatitis. Pancreas 2008; 37: 449-53.

8. Bihari D. Monitoring procalcitonin is of value in acute pancreatitis. BMJ 2004; 329: 232.

9. Mofidi R, Suttie SA, Patil PV, Ogston S, Parks RW. The value of procalcitonin at predicting the severity of acute pancreatitis and development of infected pancreatic necrosis: systematic review. Surgery 2009; 146: 72-81.

10. Woo SM, Noh MH, Kim BG, et al. Comparison of serum procalcitonin with Ranson, APACHE-II, Glasgow and Balthazar CT severity index scores in predicting severity of acute pancreatitis. Korean J Gastroenterol 2011; 58: 31-7.

11. Bezmarevic M, Mirkovic D, Soldatovic I, et al. Correlation between procalcitonin and intra-abdominal pressure and their role in prediction of the severity of acute pancreatitis. Pancreatology 2012; 12: 337-43.

12. Qu R, Ji Y, Ling Y, et al. Procalcitonin is a good tool to guide duration of antibiotic therapy in patients with severe acute pancreatitis. A randomized prospective single-center controlled trial. Saudi Med J 2012; 33: 382-7.

13. Kim BG, Noh MH, Ryu CH, et al. A comparison of the BISAP score and serum procalcitonin for predicting the severity of acute pancreatitis. Korean J Intern Med 2013; 28: 322-9.

14. Huang HL, Nie X, Cai B, et al. Procalcitonin levels predict acute kidney injury and prognosis in acute pancreatitis: a prospective study. PLoS One 2013; 8: e82250.

15. Cai Y, Ee J, Liew YX, et al. A procalcitonin-based guideline promotes shorter duration of antibiotic use safely in acute pancreatitis. J Infect 2014; 69: 412-5.

16. Banks PA, Bollen TL, Dervenis C, et al. Classification of acute pancreatitis - 2012: revision of the Atlanta classification and definitions by international consensus. Gut 2013; 62: 102-11.

17. Tenner S, Baillie J, DeWitt J, Vege SS. American College of Gastroenterology guideline: management of acute pancreatitis. Am J Gastroenterol 2013; 108: 1400-15. 
18. Qu Z, Jiang Y, Wu BQ, Duan YF, Sun ZD, Luo GH. Cystathionine-gamma-lyase inhibitor attenuates acute lung injury induced by acute pancreatitis in rats. Arch Med Sci 2014; 10: 825-9.

19. Kusnierz-Cabala B, Gurda-Duda A, Dumnicka P, et al Plasma pentraxin 3 concentrations in patients with acute pancreatitis. Clin Lab 2012; 59: 1003-8.

20. Meryk P, Dumnicka P, Kuśnierz-Cabala B, et al. [Diagnostic importance of pentraxins at the early phase of acute pancreatitis]. Przegl Lek 2013; 71: 309-13.

21. Chooklin S. Pathogenic aspects of pulmonary complications in acute pancreatitis patients. Hepatobiliary Pancreat Dis Int 2009; 8: 186-92.

22. Park BK, Chung JB, Lee JH, et al. Role of oxygen free radicals in patients with acute pancreatitis. World J Gastroenterol 2003; 9: 2266-9.

23. Lefkowitz D, Mone J, Lefkowitz S. Myeloperoxidase: the good, the bad, and the ugly. Curr Immunol Rev 2010; 6: 123-9.

24. Modrau IS, Floyd AK, Thorlacius-Ussing O. The clinical value of procalcitonin in early assessment of acute pancreatitis. Am J Gastroenterol 2005; 100: 1593-7.

25. Rau BM, Kemppainen EA, Gumbs AA, et al. Early assessment of pancreatic infections and overall prognosis in severe acute pancreatitis by procalcitonin (PCT): a prospective international multicenter study. Ann Surg 2007; 245: 745-54.

26. Staubli SM, Oertli D, Nebiker CA. Laboratory markers predicting severity of acute pancreatitis. Crit Rev Clin Lab Sci 2015; 52: 273-83.

27. Mándi Y, Farkas G, Takács T, Boda K, Lonovics J. Diagnostic relevance of procalcitonin, IL-6, and SICAM-1 in the prediction of infected necrosis in acute pancreatitis. Int J Pancreatol 2000; 28: 41-9.

28. Kylänpää-Bäck ML, Takala A, Kemppainen EA, et al. Procalcitonin, soluble interleukin-2 receptor, and soluble E-selectin in predicting the severity of acute pancreatitis. Crit Care Med 2001; 29: 63-9.

29. d'Eril GM, Merlini G, Finazzi S, Bosoni T, Barakat B, Pezzilli R. Procalcitonin is not a reliable marker for the assessment of severity in acute pancreatitis without infectious complications. Clin Chem 2000; 46: 428-30.

30. Müller C, Uhl W, Printzen G, et al. Role of procalcitonin and granulocyte colony stimulating factor in the early prediction of infected necrosis in severe acute pancreatitis. Gut 2000; 46: 233-8.

31. Rau B, Steinbach G, Gansauge F, Mayer J, Grünert A, Beger $\mathrm{H}$. The potential role of procalcitonin and interleukin 8 in the prediction of infected necrosis in acute pancreatitis. Gut 1997; 41: 832-40.

32. Ammori B, Becker K, Kite P, et al. Calcitonin precursors: early markers of gut barrier dysfunction in patients with acute pancreatitis. Pancreas 2003; 27: 239-43.

33. Ammori B, Becker K, Kite P, et al. Calcitonin precursors in the prediction of severity of acute pancreatitis on the day of admission. Br J Surg 2003; 90: 197-204. 